\title{
Drug survival and the associated predictors in South Korean patients with rheumatoid arthritis receiving tacrolimus
}

\author{
Eun-Young Park ${ }^{1}$, Seung-Geun Lee ${ }^{1}$, Eun-Kyoung Park ${ }^{1}$, Dong-Wan Koo ${ }^{1}$, Ji-Heh Park ${ }^{1}$, Geun-Tae Kim² \\ Hee-Sang $\mathrm{Tag}^{2}$, Hyun-Ok Kim³ , and Young-Sun Suh ${ }^{3}$
}

\begin{abstract}
${ }^{1}$ Division of Rheumatology, Department of Internal Medicine, Pusan National University Hospital, Pusan National University School of Medicine, Busan; ${ }^{2}$ Division of Rheumatology, Department of Internal Medicine, Kosin University College of Medicine, Busan; ${ }^{3}$ Division of Rheumatology, Department of Internal Medicine, Gyeongsang National University School of Medicine, Jinju, Korea
\end{abstract}

Received: November 29, 2015 Revised : December 21, 2015 Accepted: December 28, 2015

\section{Correspondence to}

Seung-Geun Lee, M.D.

Division of Rheumatology,

Department of Internal Medicine,

Pusan National University

Hospital, Pusan National

University School of Medicine, 179

Gudeok-ro, Seo-gu, Busan 49241,

Korea

Tel: $+82-51-240-7580$

Fax: +82-51-241-7580

E-mail: sglee@pusan.ac.kr
Background/Aims: To investigate the drug survival rate of tacrolimus (TAC) and analyze the potential predictors of this rate in patients with rheumatoid arthritis (RA) in routine care.

Methods: In this retrospective longitudinal study, we enrolled 102 RA patients treated with TAC from April 2009 to January 2014 at a tertiary center in South Korea. The causes of TAC discontinuation were classified as lack of efficacy (LOE), adverse events (AEs), and others. The drug survival rate was estimated using the Kaplan-Meier method and the predictors of this rate were identified by Coxregression analyses.

Results: TAC was discontinued in 27 of 102 RA patients (26.5\%). The overall 1-, 2-, $3-$, and 4-year TAC continuation rates were $81.8 \%, 78.4 \%, 74.2 \%$, and $69.1 \%$, respectively and the median follow-up period from the start of TAC was 32.5 months. The number of TAC discontinuations due to LOE, AEs, and others were 15 (55.6\%), $11(40.7 \%)$, and 1 (3.7\%), respectively. The baseline high disease activity was a significant risk factor for TAC discontinuation after adjusting for confounding factors (hazard ratio [HR], 2.49; 95\% confidence interval [CI], 1.16 to 5.35; $p=0.019$ ). In addition, underlying interstitial lung disease was significantly associated with TAC withdrawal due to AEs (HR, 3.49; 95\% CI, 1.06 to $11.46 ; p=0.039$ ).

Conclusions: In our study, TAC showed a good overall survival rate in patients with RA in real clinical practice. This suggests that the long-term TAC therapy has a favorable efficacy and safety profile for treating RA.

Keywords: Tacrolimus; Arthritis, rheumatoid; Drug survival; Treatment outcome; Safety

\section{INTRODUCTION}

Drug survival or retention has been reported as a composite measure of efficacy, safety, and tolerability in clinical practice $[1,2]$. Because of its chronicity and incurability, rheumatoid arthritis (RA) usually requires life-long therapy with disease modifying anti-rheu- matic drugs (DMARDs); thus, long term retention of a DMARD is an important indicator of the performance of that drug in terms of managing of RA [3]. In light with this notion, growing attention has been paid to survival rate of various drugs in patients with RA from numerous national-wide registries or observational data reflecting daily practice. Among them, tumor necrosis 
factor $\alpha$ (TNF- $\alpha$ ) inhibitors have been most extensively investigated [1-15], but relevant studies regarding tocilizumab and abatacept also have also been published in recent years $[9,13,16]$. Although more patients with RA were treated with conventional synthetic DMARDs than with biological agents in real practice, relatively little attention has been paid recently to the drug survival rate of these drugs, except for leflunomide [17-20].

Tacrolimus (TAC, FK506), a macrolide derived from Streptomyces tsukubaensis, was initially developed and used in organ transplantation [21]. It exerts an immunosuppressive action by inhibiting T-cell proliferation. After binding to the FK-binding protein, TAC inhibits calcineurin phosphatase and subsequently prevents translocation of the nuclear factor of activated T-cells which is needed for the production of cytokines such as interleukin 2 and interferon $\gamma$ [21]. Because T-cell activation has a key role in the pathogenesis of RA, randomized clinical trials for TAC monotherapy or in combination therapy with methotrexate (MTX) in patients with RA have shown the remarkable efficacy and safety [22-25]. Thus, TAC can be applied as an additional therapeutic option for RA $[21,26]$. After TAC was approved for the first time in the world for treating RA in Canada in February 2004, it was also available as a DMARD for patients with RA in Japan and South Korea in April 2005 and October 2008, respectively. Then, several observational studies regarding the tolerability and effectiveness including radiographic changes of TAC treatment in Japanese patients with RA in various clinical settings, have been published [27-30]. However, long-term follow-up data about drug survival and the associated factors among patients with RA receiving TAC are still lacking to date, especially in South Korea. Therefore, the present study aimed to investigate the drug survival rate of TAC in the treatment of RA and to analyze the potential predictors of this rate in routine clinical care.

\section{METHODS}

\section{Study design and subjects}

We conducted a retrospective longitudinal study among consecutive 102 patients with RA who were treated with TAC (Prograf, Astellas Pharma, Tokyo, Japan) and followed at least 1 year follow-up from April 2009 to Janu- ary 2014 at a university-affiliated rheumatology center in South Korea. All study subjects fulfilled the 1987 American College of Rheumatology classification criteria for RA [31] and they were followed longitudinally through reviewing of their entire medical records (inpatient and outpatient) until January 2015. The procedures of our study were approved by the Research and Ethical Review Board of the Pusan National University Hospital, which waived the need for informed patient consent (approval no.: E-2015076).

For the study subjects, the baseline clinical and laboratory parameters including their age at diagnosis, disease duration, sex, rheumatoid factor (RF) and anti-cyclic citrullinated peptide (anti-CCP) antibody status, number of previous DMARDs, concomitant medication (i.e., DMARDs, glucocorticoids, and nonsteroidal anti-inflammatory drugs), comorbid interstitial lung disease (ILD) status, swollen joint count (SJC), tender joint count (TJC), patient's general health visual analog scale score (rated from o to 100), erythrocyte sedimentation rate (ESR), and C-reactive protein (CRP) level were obtained and recorded. TAC treatment data such as the initial dosage and date of initiation and/or termination were also reviewed. Disease duration was defined as the difference between the date of diagnosis and the date of TAC initiation. ILD was defined as bibasilar fibrosis on chest radiography and/or fibrosis or ground-glass opacity on high resolution chest computed tomography in the absence of other causes. RF was assessed by a particle-enhanced immunoturbidimetric assay (range, o to $14 \mathrm{IU} / \mathrm{mL}$ ) and anti-CCP was measured using a chemiluminescent microparticle immunoassay (range, o to $5 \mathrm{U} / \mathrm{mL}$ ). The CRP level was measured by a particle-enhanced immunoturbidimetric assay (Tina-quant CRP, Roche Diagnostics, Basel, Switzerland) using a P-8oo Modular analyzer (Roche Diagnostics). The Disease Activity Score assessed by using 28-joint counts for swelling and tenderness (DAS28)-ESR score was calculated by the following formula: DAS28-ESR score $=0.56 \times \sqrt{ }$ TJC $28+0.28 \times \sqrt{ }$ SJC $28+0.70 \times \operatorname{lnESR}+0.0014 \times$ visual analog scale score [32].

A DAS28-ESR of $>5.1$ defines high disease activity; 3.2 $<$ DAS28-ESR $\leq 5.1$ is a moderate disease activity; $2.6<$ DAS28-ESR $\leq 3.2$ is a low disease activity; DAS28-ESR $\leq$ 2.6 is a remission [33].

The main outcome of the present study was TAC dis- 
continuation of any cause. The causes of TAC discontinuation were classified as follows: (1) lack of efficacy (LOE), (2) adverse events (AEs), and (3) others (e.g., patient or medical decision and miscellaneous reasons), which were judged based on the patient's medical records. The time to TAC discontinuation was calculated from the date of TAC initiation to the first treatment interruption. Interruptions were considered to be definitive when no consecutive re-introduction of TAC was recorded based on medical chart reviews [15].

\section{Statistical analysis}

Data are summarized as the mean and standard deviation (with normal distribution) or median and interquartile range (with non-normal distribution) for continuous variables, and a number of cases with percentages for categorical variables, as appropriate. The Kolmogorov-Smirnov tests were used to assess the normal distribution of data. The drug survival rates of TAC were calculated and plotted using Kaplan-Meier curves and compared using the log-rank test. To investigate the potential predictors of TAC discontinuation, we used multivariable Cox-proportional hazard regression analyses with backward model selection of demographic variables such as age at TAC initiation and sex as well as variables that had a $p \leq 0.1$ in univariable analysis. All statistical tests were two-sided and $p$ values less than 0.05 were considered statistically significant. All analyses were performed using PASW version 18.0 (SPSS Inc., Chicago, IL, USA) and STATA version 11.0 (StataCorp LP, College Station, TX, USA).

\section{RESULTS}

Table 1 shows the baseline clinical characteristics of patients with RA treated with TAC. The mean age at the initiation of TAC was $54.2 \pm 13.3$ years and the median disease duration was 34 months (range, 8 to 73). Most patients were female (77.5\%) with a positive RF or anti-CCP antibody and the frequency of ILD was $20.6 \%$. The mean DAS28-ESR score at the initiation of TAC treatment was $4.83 \pm 1.13$ and the numbers of RA patients with high, moderate and low disease activity were 38 (37.3\%), 55 (53.9\%), and 8 (7.8\%), respectively. Although five patients with RA were treated with TAC only, most study subjects
Table 1. Clinical characteristics of patients with rheumatoid arthritis receiving tacrolimus

\begin{tabular}{|c|c|}
\hline Variable & $\begin{array}{l}\text { RA patients } \\
\quad(\mathrm{n}=102)\end{array}$ \\
\hline Female sex & $79(77.5)$ \\
\hline Age at the diagnosis of RA, yr & $50.3 \pm 13.1$ \\
\hline Age at the initiation of TAC, yr & $54.2 \pm 13 \cdot 3$ \\
\hline $\begin{array}{l}\text { Disease duration at the initiation } \\
\text { of TAC, mon }\end{array}$ & $34(8-73)$ \\
\hline RF positive & $82(80.4)$ \\
\hline Anti-CCP antibody positive & $63(61.8)$ \\
\hline ILD & $21(20.6)$ \\
\hline $\mathrm{ESR}, \mathrm{mm} / \mathrm{hr}$ & $38(24-65 \cdot 5)$ \\
\hline $\mathrm{CRP}, \mathrm{mg} / \mathrm{dL}$ & $0.89(0.2-2.25)$ \\
\hline Baseline DAS28-ESR & $4.83 \pm 1.13$ \\
\hline \multicolumn{2}{|l|}{ Initial dose of TAC, mg/day } \\
\hline 1.5 & $50(49)$ \\
\hline$>1.5$ & $52(51)$ \\
\hline \multicolumn{2}{|l|}{ Last dose of TAC, mg/day } \\
\hline 1.5 & $36(35 \cdot 3)$ \\
\hline$>1.5$ & $66(64.7)$ \\
\hline TAC monotherapy & $5(4 \cdot 9)$ \\
\hline \multicolumn{2}{|l|}{ Concomitant DMARDs } \\
\hline MTX & $81(79.4)$ \\
\hline MTX dose, mg/wk & $10(6.25-12.5)$ \\
\hline HCQ & $24(23 \cdot 5)$ \\
\hline SSZ & $3(2.9)$ \\
\hline Concomitant NSAIDs & $90(88.2)$ \\
\hline Concomitant glucocorticoids & $98(96.1)$ \\
\hline \multicolumn{2}{|l|}{ No. of previous DMARDs } \\
\hline 0 & $7(6.9)$ \\
\hline 1 & $35(34 \cdot 3)$ \\
\hline 2 & $47(46.1)$ \\
\hline$\geq 3$ & $13(12.7)$ \\
\hline
\end{tabular}

Values are presented as number $(\%)$, mean $\pm \mathrm{SD}$, or median (interquartile range).

RA, rheumatoid arthritis; TAC, tacrolimus; RF, rheumatoid factor; CCP, cyclic citrullinated peptide; ILD, interstitial lung disease; ESR, erythrocyte sedimentation rate; CRP, C-reactive protein; DAS28, Disease Activity Score assessed by using 28-joint counts for swelling and tenderness; DMRAD, disease modifying anti-rheumatic drug; MTX, methotrexate; HCQ, hydroxychloroquine; SSZ, sulfasalazine; NSAID, nonsteroidal anti-inflammatory drug. 


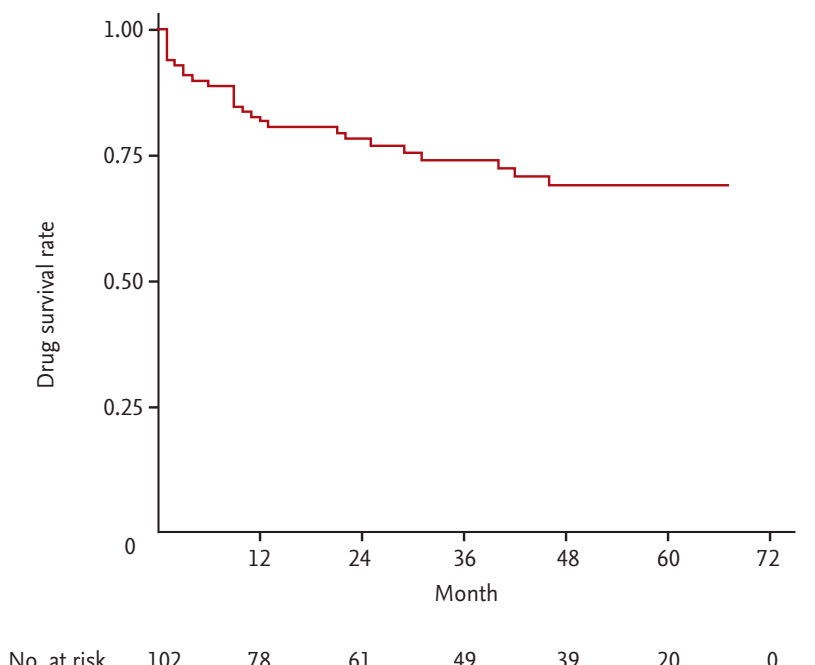

Figure 1. Kaplan-Meier survival curve of tacrolimus in patients with rheumatoid arthritis.

were receiving TAC plus DMARDs combination therapy; the most common concomitant DMARDs was MTX. In addition, most patients with RA were treated with one or more DMARDs before TAC therapy, but TAC was started at the diagnosis of RA in seven subjects (6.9\%). The mean initial and last dose of TAC during the study period were $1.76 \pm 0.25$ and $1.93 \pm 0.39 \mathrm{mg}$, respectively.

Twenty-seven patients $(26.5 \%)$ discontinued TAC treatment among 102 patients with RA. The overall 1-, 2-, 3-, and 4-year TAC continuation rates were $81.8 \%, 78.4 \%$, $74.2 \%$, and $69.1 \%$, respectively and the highest discontinuation rate was within the first year after TAC initiation (Fig. 1). The median follow-up period from the start of TAC was 32.5 months (range, 12 to 55.3). As shown in Fig. 2, the drug survival rate of patients with a high disease activity was significantly lower than those with a low to moderate disease activity $(p=0.014)$. The most common cause of TAC discontinuation was LOE (55.6\%), whereas AEs resulting in TAC discontinuation occurred in 11 patients (40.7\%) with RA (Table 2). Four patients with RA stopped TAC because of gastrointestinal disorders including nausea and diarrhea. Cardiopulmonary disorders leading to TAC withdrawal such as ILD exacerbation and angina pectoris occurred in three patients. Preceding bronchitis, especially, was regarded to have triggered ILD exacerbation in two patients. Allergic reaction, peripheral neuropathy, and hyperkalemia

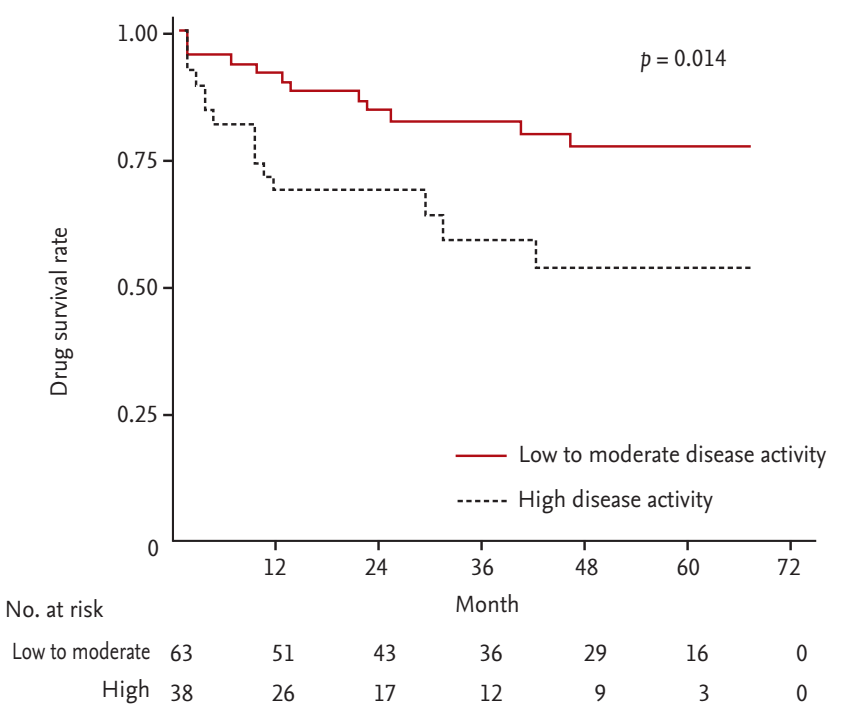

Figure 2. Comparisons of the discontinuation rate of tacrolimus according to the disease activity of the patients.

Table 2. Number and causes of tacrolimus discontinuation in patients with rheumatoid arthritis

\begin{tabular}{lc}
\hline Variable & $\begin{array}{c}\text { TAC discontinuation } \\
(\mathrm{n}=27)\end{array}$ \\
\hline Lack of efficacy & $15(55.6)$ \\
\hline Adverse events & $11(40.7)$ \\
Gastrointestinal disorder & $4(14.8)$ \\
Cardiopulmonary disorder & $3(11.1)$ \\
Angina pectoris & $1(3.7)$ \\
ILD exacerbation & $2(7.4)$ \\
Allergic reaction & $2(7.4)$ \\
Peripheral neuropathy & $1(3.7)$ \\
Hyperkalemia & $1(3.7)$ \\
Others (patients' decision) & $1(3.7)$ \\
\hline
\end{tabular}

Values are presented as number (\%).

TAC, tacrolimus; ILD, interstitial lung disease.

developed in two, one, and one subjects, respectively. However, all AEs were mild or moderate and resolved after discontinuing TAC and subsequent appropriate management. In addition, TAC was not discontinued owing to renal impairment or glucose metabolism abnormality. One patient with RA stopped TAC 3 months after initiation because of the cost of the medicine.

Table 3 summarizes several significant predictors for TAC discontinuation in patients with RA using 
Table 3. Cox-proportional hazard regression analyses with backward model selection for tacrolimus discontinuation according to causes

\begin{tabular}{|c|c|c|c|c|}
\hline \multirow{2}{*}{ Variable } & \multicolumn{2}{|c|}{ Univariable } & \multicolumn{2}{|c|}{ Multivariable } \\
\hline & $\operatorname{HR}(95 \% \mathrm{CI})$ & $p$ value & $\operatorname{HR}(95 \% \mathrm{CI})$ & $p$ value \\
\hline \multicolumn{5}{|l|}{ TAC discontinuation due to any cause } \\
\hline Baseline DAS28-ESR > 5.1 & $2.49(1.16-5.35)$ & 0.019 & $2.49(1.16-5.35)$ & 0.019 \\
\hline Age at the TAC initiation $<60 \mathrm{yr}$ & $1.63(0.69-3.86)$ & 0.264 & & \\
\hline Initial dose of TAC > $1.5 \mathrm{mg} /$ day & $1.67(0.77-3.66)$ & 0.197 & & \\
\hline Last dose of TAC > $1.5 \mathrm{mg} /$ day & $1.34(0.59-3.07)$ & 0.489 & & \\
\hline Concomitant MTX treatment & $1.08(0.41-2.87)$ & 0.87 & & \\
\hline No. of previous DMARDs & $0.91(0.57-1.43)$ & 0.681 & & \\
\hline Disease duration $>24$ mon & $0.71(0.34-1.52)$ & 0.381 & & \\
\hline Male sex & $1.14(0.46-2.83)$ & 0.777 & & \\
\hline ILD & $0.94(0.36-2.49)$ & 0.908 & & \\
\hline RF positive & $0.65(0.24-1.75)$ & 0.393 & & \\
\hline Anti-CCP antibody positive & $0.95(0.35-2.56)$ & 0.918 & & \\
\hline \multicolumn{5}{|l|}{ TAC discontinuation due to LOE } \\
\hline Baseline DAS28-ESR > 5.1 & $3.04(1.07-8.58)$ & 0.036 & $3.55(1.25-10.09)$ & 0.017 \\
\hline Age at the TAC initiation $<60 \mathrm{yr}$ & $3.65(0.82-16.17)$ & 0.089 & $4.46(1.01-19.91)$ & 0.049 \\
\hline Initial dose of TAC > $1.5 \mathrm{mg} /$ day & $2.66(0.84-8.37)$ & 0.094 & & \\
\hline Last dose of TAC > 1.5 mg/day & $1.52(0.48-4.8)$ & 0.472 & & \\
\hline Concomitant MTX treatment & $28.28(0.12-6,819.58)$ & 0.232 & & \\
\hline No. of previous DMARDs & $0.8(0.43-1.48)$ & 0.478 & & \\
\hline Disease duration $>24$ mon & $0.75(0.27-2.07)$ & 0.581 & & \\
\hline Male sex & $1.01(0.29-3.59)$ & 0.983 & & \\
\hline ILD & $0.04(0-8.84)$ & 0.236 & & \\
\hline RF positive & $1.03(0.23-4.61)$ & 0.968 & & \\
\hline Anti-CCP antibody positive & $1.53(0.34-6.9)$ & 0.581 & & \\
\hline \multicolumn{5}{|l|}{ TAC discontinuation due to AEs } \\
\hline Baseline DAS28-ESR > 5.1 & $1.65(0.5-5.44)$ & 0.411 & & \\
\hline Age at the TAC initiation $<60 \mathrm{yr}$ & $1.03(0.3-3.51)$ & 0.967 & & \\
\hline Initial dose of TAC > $1.5 \mathrm{mg} /$ day & $1.22(0.37-4.01)$ & 0.742 & & \\
\hline Last dose of TAC > 1.5 mg/day & $1.57(0.42-5.92)$ & 0.508 & & \\
\hline Concomitant MTX treatment & $0.29(0.9-0.98)$ & 0.045 & & \\
\hline No. of previous DMARDs & $1.02(0.5-2.1)$ & 0.961 & & \\
\hline Disease duration $>24$ mon & $0.8(0.24-2.63)$ & 0.713 & & \\
\hline Male sex & $1.47(0.39-5.54)$ & 0.571 & & \\
\hline ILD & $3.49(1.06-11.46)$ & 0.039 & $3.49(1.06-11.46)$ & 0.039 \\
\hline RF positive & $0.34(0.09-1.36)$ & 0.128 & & \\
\hline Anti-CCP antibody positive & $0.48(0.12-1.94)$ & 0.307 & & \\
\hline
\end{tabular}

HR, hazard ratio; CI, confidence interval; TAC, tacrolimus; DAS28-ESR, Disease Activity Score assessed by using 28-joint counts for swelling and tenderness-erythrocyte sedimentation rate; MTX, methotrexate; DMRAD, disease modifying antirheumatic drug; ILD, interstitial lung disease; RF, rheumatoid factor; CCP, cyclic citrullinated peptide; LOE, lack of efficacy; $\mathrm{AE}$, adverse event. 
Cox-proportional regression models. The baseline high disease activity (hazard ratio [HR], 2.49; 95\% confidence interval [CI], 1.16 to $5.35 ; p=0.019$ ) was the independent risk factor for TAC withdrawal due to any cause after adjusting for confounding factors. However, the initial or last dose of TAC, concomitant MTX treatment, number of previous DMARDs, disease duration and RF or anti-CCP antibody positivity were not related to drug survival of TAC. In addition, concomitant sulfasalazine or hydroxychloroquine therapy and the dose of MTX did not have a significant association with TAC discontinuation (data not shown). We also investigated predictors for cause-specific TAC discontinuation. The baseline high disease activity and age at the TAC initiation less than 60 years were significant predictors for TAC withdrawal due to LOE. Of interest, RA patients with ILD showed significantly lower TAC retention due to AEs after adjusting for confounding factors (HR, 3.49; $95 \%$ CI, 1.06 to $11.46 ; p=0.039)$. However, age at the TAC initiation, initial dose of TAC and concomitant MTX use did not show the statistically significant association with the increased TAC discontinuation rate due to AEs. In addition, the number of previous DMARDs was not related to TAC survival due to any cause, LOE, and AEs (data not shown).

\section{DISCUSSION}

The present study investigated the long term retention rate of TAC used for patients with RA in clinical practice. Our study demonstrated that the drug survival rates of TAC after 1, 2, 3, and 4 year were 81.8\%, 78.4\%, 74.2\%, and $69.1 \%$, respectively with a median follow-up duration of 32.5 months in patients with RA. The highest rate of TAC discontinuation occurred in the first year. The baseline high disease activity, defined as a DAS28-ESR of > 5.1, was a significant predictor for TAC discontinuation. The two main reasons for TAC withdrawal were LOE and AEs, and more patients with RA discontinued TAC due to LOE (55.6\%) than due to AEs (40.7\%). The age at the TAC initiation less than 60 years and the baseline high disease activity were associated with TAC discontinuation due to LOE, whereas underlying ILD was a risk factor for worse survival of TAC due to AEs.

Drug survival is known to be a surrogate marker of the long-term efficacy and safety of a drug in real clinical practice, but patient's and physician's satisfaction with the treatment, cost, convenience, and availability of therapeutic alternatives can also influence the drug retention rate. Because RA requires lifelong treatment to control inflammation and joint damage, DMARDs with a high retention may be an optimal choice in managing RA. In previous studies, the 1 and 2 year survival rate of anti-TNF- $\alpha$ agents in patients with RA approximately ranged from approximately $60 \%$ to $80 \%$ and $40 \%$ to $70 \%$, respectively [1-15]. When compared with TNF- $\alpha$ inhibitors, non-TNF- $\alpha$ biologic agents such as tocilizumab and abatacept showed similar rates of retention in the observational studies $[9,13,16]$. With respect to conventional synthetic DMARDs, the 2-year continuation rate of leflunomide varies from $44.7 \%$ to $71 \%$, but two recent studies conducted in the early 2010 S demonstrated a better retention rate with leflunomide than that found in previous data [18-20]. Our finding of a 2-year TAC survival of $78.4 \%$ in RA patients seems to be comparable to that of leflunomide or even biologic agents. Although comparing the survival rate between TAC and other DMARDs should be performed cautiously because of the difference in the baseline clinical features, including the disease activity between the previous studies and ours, our data suggests that TAC has a good long term effectiveness and tolerability of TAC in the treatment of RA in real clinical practice. However, further investigations are needed to compare the persistence rate between TAC and other agents.

To our knowledge, only one previous study has calculated the survival figures for TAC in patients with RA. Ogasawara et al. [28] reported that the 1 and 2 year survival rates of TAC in 115 Japanese patients with RA were $57.9 \%$ and $48.9 \%$, respectively, which were lower than those in our study. The baseline disease activity, considered as a risk factor for TAC discontinuation in our study, was comparable between the Ogasawara et al. [28]'s study and ours. Thus, the discrepancy of TAC survival between both studies may be related to the difference in ethnicity among the study subjects, practice guidelines or the incidence of AEs leading to TAC withdrawal. Compared with our data, Ogasawara et al. [28]'s study had a shorter follow-up period and did not evaluate the cause specific survival of TAC. Hence, our study provided more comprehensive data on TAC survival in 
clinical practice. Kitahama et al. [27] and Kanzaki et al. [29] also assessed the retention of TAC in Japanese patients with RA. However, these studies did not show the exact figures for the survival rate and cannot be generalized because of short follow-up period (12 months) and the small sample size $(n=24)$ in Kitahama et al. [27]'s and Kanzaki et al. [29]'s studies, respectively.

In our study, the most common cause of TAC discontinuation was LOE (55.6\%), followed by AEs (40.7\%). Similarly, previous observational data showed that LOE was the more common cause of discontinuation of anti-TNF- $\alpha$ agents than AEs in patients with RA $[3,4,14]$, although some studies have reported contradictory results $[8,12]$. Gomez-Reino et al. [7] demonstrated that patients with RA starting TNF- $\alpha$ inhibitors more recently had a higher risk of discontinuation due to LOE, compared with earlier studies, whereas the survival rate due to AEs had remained stable. Because of the adaptation of more aggressive treatment strategies, development of newer efficacious biologic agents and higher expectation about the response to therapy or remission, switching between biologic agents occurred more frequently in recent years according to previous data [7,34]. This idea was consistent with our finding that LOE was the main cause of TAC discontinuation, considering that most of our RA patients had an inadequate response to one or more DMARDs before TAC therapy.

Since our study included more than 100 patients with RA long follow-up period, investigating the predictors for TAC survival was possible. The baseline high disease activity was an independent risk factor for TAC discontinuation in the present study. Furthermore, in the cause-specific analyses, a high disease activity was associated with TAC withdrawal due to LOE, but not AEs. We can infer from this finding that RA patients with a high disease activity would have had more of a chance to discontinue TAC, especially due to an inadequate treatment response. The relationship between a higher DAS2 8 and worse survival of anti-TNF- $\alpha$ agents was also observed in the previous studies $[4,7]$. In addition, previous data showed a dose-dependent response to TAC in patients with RA [22], but the initial or last dose of TAC was not significantly associated with drug survival in our study. As the initial or last dose of TAC usually ranged from 1.5 to $2.5 \mathrm{mg}$, the dose-dependent manner of TAC survival was not shown in our data. Of interest, age at the TAC initiation less than 60 years was a significant predictor for worse TAC survival due to LOE in our study, which was contrary to the results of previous studies regarding the survival of anti-TNF- $\alpha$ agents and leflunomide $[7,10,19]$. The inverse relationship between the baseline age and TAC discontinuation in our study may be explained by the higher expectation for the treatment outcome in younger patients with RA or the difference of TAC efficacy according to age. However, because of the lack of data concerning the association between the efficacy of TAC and the baseline age, this finding remains controversial and needs to be further confirmed by additional studies. In addition, concomitant MTX treatment was reported to increase the retention rate of anti-TNF- $\alpha$ agents and leflunomide $[2,6,19]$, but these trends were not found in the present study.

Another significant finding in our study was that underlying ILD was related to TAC withdrawal due to AEs. Some of the most frequently prescribed DMARDs such as MTX, leflunomide, and anti-TNF- $\alpha$ agents have been implicated with pulmonary toxicities including ILD exacerbation [35]. Otherwise, calcineurin antagonists can be used as a therapeutic option to treat ILD associated with connective tissue disorders including idiopathic inflammatory myopathies [35,36]. Thus, it has been presumed that TAC may be safer in patients with RA-ILD than other DMARDs such as MTX. However, recent case reports raised a possibility of TAC induced pulmonary injury in patients with RA, particularly with pre-existing ILD [37]. Additionally, in the post-marketing surveillance of TAC in 3,267 Japanese patients with RA, ILD was reported to occur in 17 cases (0.5\%) [38]. Taken together with our finding, careful attention needed to be paid to RA patients with ILD who are receiving TAC.

We note a number of potential limitations to our study. First, we retrieved the drug survival data for TAC only; thus, direct comparisons between TAC and other DMARDs were not possible. However, a majority of previous studies on drug survival have analyzed a single agent, except for studies on TNF- $\alpha$ inhibitors [1619,27,29]. Thus, we think that our data also provide useful clinical information regarding the long-term retention rate of TAC. As mentioned above, further research is needed to compare the persistence rate between TAC and other DMARDs. Second, unmeasured confounding factors such as the clinician's personal preference 
and economic burden of TAC cannot be completely excluded. In South Korea, TAC is the most expensive conventional DMARD and it costs approximately \$3.30 per $1 \mathrm{mg}$ capsule. In general, patients have a tendency to have higher expectations for more expensive drugs, which may have affected our results. Third, because of the retrospective study design, some information such as the follow-up disease activity after TAC therapy could not be retrieved. Fourth, because of the small number of subjects who were treated with TAC monotherapy, it was impossible to compare the drug survival rate between TAC monotherapy and combination treatment with other DMARDs. This may have also contributed to the lack of statistical association between concomitant MTX treatment and better TAC survival in our study. Lastly, we recruited all of our patients with RA from a single tertiary hospital, which could have resulted in a selection bias.

In summary, TAC showed a good overall survival rate in patients with RA in real clinical practice. This suggests that the long-term TAC therapy has a favorable efficacy and safety profile for treating RA. The baseline high disease activity was a risk factor for TAC discontinuation, similarly with anti-TNF- $\alpha$ agents. In addition, underlying ILD was significantly associated with TAC withdrawal due to AEs. Therefore, careful evaluation of pulmonary toxicities may be needed in patients with RA receiving TAC. Although TAC has not been widely used as DMARDs in patients with RA in many Western countries, TAC may be a good option for managing RA in terms of long-term drug survival based on our data.

\section{KEY MESSAGE}

1. Tacrolimus (TAC) showed a good overall survival rate in patients with rheumatoid arthritis (RA) in real clinical practice, suggesting the favorable long-term efficacy and tolerability of TAC for treating RA.

2. The baseline high disease activity was a significant risk factor for TAC discontinuation.

3. Underlying interstitial lung disease was significantly associated with TAC withdrawal due to adverse events.

\section{Conflict of interest}

No potential conflict of interest relevant to this article was reported.

\section{Acknowledgments}

This work was supported by clinical research grant from Pusan National University Hospital 2015. We specially thank the late Professor Sung-Il Kim who devoted himself to education, research, and patient care in Division of Rheumatology, Department of Internal Medicine, Pusan National University School of Medicine (1963 to 2011).

\section{REFERENCES}

1. Greenberg JD, Reed G, Decktor D, et al. A comparative effectiveness study of adalimumab, etanercept and infliximab in biologically naive and switched rheumatoid arthritis patients: results from the US CORRONA registry. Ann Rheum Dis 2012;71:1134-1142.

2. Neovius M, Arkema EV, Olsson H, et al. Drug survival on TNF inhibitors in patients with rheumatoid arthritis comparison of adalimumab, etanercept and infliximab. Ann Rheum Dis 2015;74:354-360.

3. Marchesoni A, Zaccara E, Gorla R, et al. TNF-alpha antagonist survival rate in a cohort of rheumatoid arthritis patients observed under conditions of standard clinical practice. Ann N Y Acad Sci 2009;1173:837-846.

4. Du Pan SM, Dehler S, Ciurea A, et al. Comparison of drug retention rates and causes of drug discontinuation between anti-tumor necrosis factor agents in rheumatoid arthritis. Arthritis Rheum 2009;61:560-568.

5. Hetland ML, Christensen IJ, Tarp U, et al. Direct comparison of treatment responses, remission rates, and drug adherence in patients with rheumatoid arthritis treated with adalimumab, etanercept, or infliximab: results from eight years of surveillance of clinical practice in the nationwide Danish DANBIO registry. Arthritis Rheum 2010;62:22-32.

6. Kievit W, Fransen J, Adang EM, et al. Long-term effectiveness and safety of TNF-blocking agents in daily clinical practice: results from the Dutch Rheumatoid Arthritis Monitoring register. Rheumatology (Oxford) 2011;50:196203.

7. Gomez-Reino JJ, Rodriguez-Lozano C, Campos-Fernandez 
$\mathrm{C}$, et al. Change in the discontinuation pattern of tumour necrosis factor antagonists in rheumatoid arthritis over 10 years: data from the Spanish registry BIOBADASER 2.0. Ann Rheum Dis 2012;71:382-385.

8. Markatseli TE, Alamanos Y, Saougou I, Voulgari PV, Drosos AA. Survival of TNF-alpha antagonists in rheumatoid arthritis: a long-term study. Clin Exp Rheumatol 2012;30:31-38.

9. Sakai R, Tanaka M, Nanki T, et al. Drug retention rates and relevant risk factors for drug discontinuation due to adverse events in rheumatoid arthritis patients receiving anticytokine therapy with different target molecules. Ann Rheum Dis 2012;71:1820-1826.

10. Yang CT, Kuo CF, Luo SF, Yu KH. Discontinuation of anti-TNF- $\alpha$ therapy in a Chinese cohort of patients with rheumatoid arthritis. Clin Rheumatol 2012;31:1549-1557.

11. Scire CA, Caporali R, Sarzi-Puttini P, et al. Drug survival of the first course of anti-TNF agents in patients with rheumatoid arthritis and seronegative spondyloarthritis: analysis from the MonitorNet database. Clin Exp Rheumatol 2013;31:857-863.

12. Biggioggero M, Favalli EG. Ten-year drug survival of antiTNF agents in the treatment of inflammatory arthritides. Drug Dev Res 2014;75 Suppl 1:S38-S41.

13. Hirabara S, Takahashi N, Fukaya N, et al. Clinical efficacy of abatacept, tocilizumab, and etanercept in Japanese rheumatoid arthritis patients with inadequate response to anti-TNF monoclonal antibodies. Clin Rheumatol 2014;33:1247-1254.

14. Kang JH, Park DJ, Lee JW, et al. Drug survival rates of tumor necrosis factor inhibitors in patients with rheumatoid arthritis and ankylosing spondylitis. J Korean Med Sci 2014;29:1205-1211.

15. Frazier-Mironer A, Dougados M, Mariette X, et al. Retention rates of adalimumab, etanercept and infliximab as first and second-line biotherapy in patients with rheumatoid arthritis in daily practice. Joint Bone Spine 2014;81:352-359.

16. Forsblad-d'Elia H, Bengtsson K, Kristensen LE, Jacobsson LT. Drug adherence, response and predictors thereof for tocilizumab in patients with rheumatoid arthritis: results from the Swedish biologics register. Rheumatology (Oxford) 2015;54:1186-1193.

17. Bettembourg-Brault I, Gossec L, Pham T, Gottenberg JE, Damiano J, Dougados M. Leflunomide in rheumatoid arthritis in daily practice: treatment discontinuation rates in comparison with other DMARDs. Clin Exp Rheumatol 2006;24:168-171.

18. Jagoda JS, Rajapakse CN. Leflunomide in clinical practice: a retrospective observational study on use of leflunomide in New Zealand. Int J Rheum Dis 2011;14:340-344.

19. Rodriguez-Rodriguez L, Jover-Jover JA, Fontsere O, et al. Leflunomide discontinuation in rheumatoid arthritis and influence of associated disease-modifying antirheumatic drugs: a survival analysis. Scand J Rheumatol 2013;42:433-436.

20. Aletaha D, Stamm T, Kapral T, et al. Survival and effectiveness of leflunomide compared with methotrexate and sulfasalazine in rheumatoid arthritis: a matched observational study. Ann Rheum Dis 2003;62:944-951.

21. Dutta S, Ahmad Y. The efficacy and safety of tacrolimus in rheumatoid arthritis. Ther Adv Musculoskelet Dis 2011;3:283-291.

22. Furst DE, Saag K, Fleischmann MR, et al. Efficacy of tacrolimus in rheumatoid arthritis patients who have been treated unsuccessfully with methotrexate: a six-month, double-blind, randomized, dose-ranging study. Arthritis Rheum 2002;46:2020-2028.

23. Yocum DE, Furst DE, Kaine JL, et al. Efficacy and safety of tacrolimus in patients with rheumatoid arthritis: a double-blind trial. Arthritis Rheum 2003;48:3328-3337.

24. Kondo H, Abe T, Hashimoto H, et al. Efficacy and safety of tacrolimus (FK506) in treatment of rheumatoid arthritis: a randomized, double blind, placebo controlled dosefinding study. J Rheumatol 2004;31:243-251.

25. Kawai S, Takeuchi T, Yamamoto K, Tanaka Y, Miyasaka N. Efficacy and safety of additional use of tacrolimus in patients with early rheumatoid arthritis with inadequate response to DMARDs: a multicenter, double-blind, parallel-group trial. Mod Rheumatol 2011;21:458-468.

26. Lee YH, Woo JH, Choi SJ, Ji JD, Bae SC, Song GG. Tacrolimus for the treatment of active rheumatoid arthritis: a systematic review and meta-analysis of randomized controlled trials. Scand J Rheumatol 2010;39:271-278.

27. Kitahama M, Okamoto H, Koseki Y, et al. Efficacy and safety of tacrolimus in 101 consecutive patients with rheumatoid arthritis. Mod Rheumatol 2010;20:478-485.

28. Ogasawara M, Tamura N, Kageyama M, et al. Single-center, retrospective analysis of efficacy and safety of tacrolimus as a second-line DMARD in combination therapy and the risk factors contributing to adverse events in 115 patients with rheumatoid arthritis. Clin Rheumatol 
2012;31:251-257.

29. Kanzaki T, Kawahata K, Kanda H, et al. Long-term therapeutic effects and safety of tacrolimus added to methotrexate in patients with rheumatoid arthritis. Rheumatol Int 2013;33:871-877.

30. Motomura H, Matsushita I, Seki E, Mine H, Kimura T. Inhibitory effect of tacrolimus on progression of joint damage in patients with rheumatoid arthritis. Int J Rheum Dis 2014;17:749-754.

31. Arnett FC, Edworthy SM, Bloch DA, et al. The American Rheumatism Association 1987 revised criteria for the classification of rheumatoid arthritis. Arthritis Rheum 1988;31:315-324.

32. Prevoo ML, van 't Hof MA, Kuper HH, van Leeuwen MA, van de Putte LB, van Riel PL. Modified disease activity scores that include twenty-eight-joint counts: development and validation in a prospective longitudinal study of patients with rheumatoid arthritis. Arthritis Rheum 1995;38:44-48.

33. van Gestel AM, Haagsma CJ, van Riel PL. Validation of rheumatoid arthritis improvement criteria that include simplified joint counts. Arthritis Rheum 1998;41:18451850.

34. Yazici Y, Krasnokutsky S, Barnes JP, Hines PL, Wang J, Rosenblatt L. Changing patterns of tumor necrosis factor inhibitor use in 9074 patients with rheumatoid arthritis. J Rheumatol 2009;36:907-913.

35. Gutsche M, Rosen GD, Swigris JJ. Connective tissue disease-associated interstitial lung disease: a review. Curr Respir Care Rep 2012;1:224-232.

36. Kobayashi A, Okamoto H. Treatment of interstitial lung diseases associated with connective tissue diseases. Expert Rev Clin Pharmacol 2012;5:219-227.

37. Koike R, Tanaka M, Komano Y, et al. Tacrolimus-induced pulmonary injury in rheumatoid arthritis patients. Pulm Pharmacol Ther 2011;24:401-406.

38. Takeuchi T, Kawai S, Yamamoto K, Harigai M, Ishida K, Miyasaka N. Post-marketing surveillance of the safety and effectiveness of tacrolimus in 3,267 Japanese patients with rheumatoid arthritis. Mod Rheumatol 2014;24:8-16. 